\title{
Probabilidade de eventos Tromboembólicos em pacientes com COVID-19: patogênese e profilaxia
}

\author{
Probability of Thromboembolic events in patients with COVID-19: pathogenesis and prophylaxis
}

Probabilidad de eventos Tromboembólicos en pacientes con COVID-19: patogénesis y profilaxis

Vitor Augusto Lima do Vale ${ }^{1 *}$, Jéssica Brambati Martins ${ }^{1}$, Júlia Eduarda Queiroz Grossi ${ }^{1}$, Pamela Andrea Alves Durães ${ }^{1}$, Raquel Moret Henrique Campos ${ }^{1}$, Thaís Maia Ribeiro ${ }^{1}$, Vitor Avelar Silvestre ${ }^{1}$, Fellipe Julião da Silva Reis ${ }^{2}$.

\section{RESUMO}

Objetivo: Analisar, por meio de uma revisão narrativa, os mecanismos fisiopatológicos que predispõem à ocorrência de eventos trombóticos e tromboembólicos em pacientes com Covid-19 e a necessidade de tromboprofilaxia nessas situações. Revisão bibliográfica: A Covid-19 pode transcorrer com sequelas pósinfecção, destacando-se os eventos trombóticos, responsáveis por elevada mortalidade. A patogênese exata da trombose nesses pacientes não é totalmente esclarecida, mas sabe-se que a doença promove resposta inflamatória exacerbada, a qual cursa com endotelite, hipercoagulabilidade, redução da fibrinólise e hipoxemia. A coagulação intravascular disseminada, à qual estão sujeitos os pacientes infectados, também contribui para o surgimento de tromboembolismo arterial e venoso. Portanto, a Covid-19 predispõe ao surgimento de outras afecções vasculares, como tromboembolismo pulmonar e acidente vascular encefálico, além da doença de Kawasaki em crianças. No intuito de prevenir esses eventos, recomenda-se o uso de heparina de baixo peso molecular em dose profilática, associado às terapêuticas mecânicas, tanto no ambiente hospitalar, como na atenção primária. Considerações finais: A patogênese da Covid-19 ainda deve ser elucidada. Diante da gravidade dos eventos trombóticos da doença, propõe-se teorias baseadas na existência de múltiplos fatores favoráveis ao desenvolvimento desta condição. Diante disto, a profilaxia antitrombótica, atua como fator significante na prevenção de fenômenos pró-coagulantes.

Palavras-chave: Coronavírus, COVID-19, Embolia pulmonar, Embolia e trombose, Trombose.

\section{ABSTRACT}

Objective: To analyze, through a narrative review, the pathophysiological mechanisms that predispose the occurrence of thrombotic and thromboembolic events in patients with Covid-19 and the need for thromboprophylaxis in these situations Bibliographic review: Covid-19 may cause post-infection sequelae, especially thrombotic events, responsible for high mortality. The exact pathogenesis of thrombosis in these patients is not fully understood, but it is known that the disease promotes an exacerbated inflammatory response, which courses with endothelitis, hypercoagulability, reduced fibrinolysis and hypoxemia. Disseminated intravascular coagulation, to which infected patients are subjected, also contributes to the onset of arterial and venous thromboembolism. Therefore, Covid-19 eventually predisposes to the emergence of other vascular disorders, such as pulmonary thromboembolism and stroke, in addition to Kawasaki disease in children. In order to prevent these events, the use of low-molecular-weight heparin in a prophylactic dose is recommended, associated with mechanical therapies, both in hospitals and primary care. Final considerations: The pathogenesis of Covid-19 is still to be elucidated. Given the severity of the disease's

\footnotetext{
${ }^{1}$ Pontifícia Universidade Católica de Minas Gerais (PUC Minas), Betim - MG.

*E-mail: vitoravale@gmail.com

2 Hospital das Clínicas de Minas Gerais, Belo Horizonte - MG.
} 
thrombotic events, theories based on the existence of multiple factors that favor the development of this condition are proposed. Therefore, antithrombotic prophylaxis acts as a significant factor in preventing procoagulant phenomena.

Key words: Coronavirus, COVID-19, Pulmonar embolism, Embolism and trombosis, Thrombosis.

\section{RESUMEN}

Objetivo: Analizar, a través de uma revisión narrativa, los mecanismos fisiopatológicos que predisponen a la ocurrencia de eventos trombóticos y tromboembólicos en pacientes con Covid-19 y la necesidad de tromboprofilaxis en estas situaciones. Revisión bibliográfica: Covid-19 puede tener secuelas posteriores a la infección, especialmente eventos trombóticos, responsables por una alta mortalidad. La patogenia exacta de la trombosis en estos pacientes no se comprende completamente, pero se sabe que la enfermedad promueve una respuesta inflamatoria exacerbada, que cursa con endotelitis, hipercoagulabilidad, fibrinólisis reducida e hipoxemia. La coagulación intravascular diseminada, a la que se someten los pacientes infectados, también contribuye a la aparición de tromboembolismo arterial y venoso. Por lo tanto, Covid-19 eventualmente predispone a la aparición de otros trastornos vasculares, como tromboembolismo pulmonar y accidente cerebrovascular, además de la enfermedad de Kawasaki en los niños. Para prevenir estos eventos se recomienda el uso de heparina de bajo peso molecular en dosis profiláctica, asociada a terapias mecánicas, tanto en el ámbito hospitalario como en atención primaria. Consideraciones finales: La patogenia de Covid19 queda por dilucidar. Dada la gravedad de los eventos trombóticos de la enfermedad, se proponen teorías basadas en la existencia de múltiples factores que favorecen el desarrollo de esta condición. Por tanto, la profilaxis antitrombótica actúa como un factor significativo en la prevención de fenómenos procoagulantes.

Palabras-clave: Coronavirus, COVID-19, Embolia pulmonar, Embolia y trombosis, Trombosis.

\section{INTRODUÇÃO}

A doença causada pelo vírus SARS-CoV-2, nomeada Covid-19, pela Organização Mundial de Saúde (OMS), foi identificada, pela primeira vez, em Wuhan, na China, ao final do ano de 2019, atingindo proporções globais no início de 2020 (ALI MAM e SPINLER SA, 2021). Na América do Sul, o Brasil foi o primeiro país a relatar um caso confirmado de infecção por esse vírus e, desde então, apresenta um contexto epidemiológico complexo, com 7.675.973 casos notificados até o final do ano de 2020 (BRASIL, 2021; LOBO AP, et al., 2020). Nesse contexto, foram identificados, até o dia 23 de Junho de 2021, 18.169 .881 casos e 507.109 óbitos confirmados, com taxa de letalidade de 2,8\%. Além disso, foram registrados 16.483 .635 pacientes recuperados e 1.179.137 em acompanhamento (BRASIL, 2021).

O SARS-CoV-2 é um vírus de Ácido Ribonucleico (RNA) que infecta a célula humana através da ligação enzima-receptor ACE2, correspondente à enzima conversora de angiotensina 2, a qual possui grande expressão nas células alveolares dos pulmões e nos tecidos cardíaco e vascular (MESA JEG, et al., 2021). Observou-se que os pacientes que apresentam sintomatologia, mais comumente, se manifestam com sinais e sintomas inespecíficos, como o mal-estar geral, a artromialgia, a astenia, a tosse, a febre, a cefaleia, a odinofagia, a anosmia e a ageusia (BELTRÁN MB, et al., 2021). Ademais, as evoluções desfavoráveis da Covid-19, por exemplo a Síndrome Respiratória Aguda Grave (SRAG), foram identificadas, em especial, nos pacientes idosos, do sexo masculino, com doenças cardiovasculares e/ou metabólicas, em que se inclui, principalmente, hipertensão arterial sistêmica, diabetes mellitus, dislipidemia, Doença Pulmonar Obstrutiva Crônica (DPOC) e obesidade (BRANDÃO SCS, et al., 2020; CUNHA MJS, et al., 2021).

Apesar de a SRAG ser uma das principais manifestações da Covid-19, a afinidade do vírus por tecidos extrapulmonares permite que os pacientes apresentem sintomatologias não respiratórias, dentre elas, os acometimentos cardiovasculares, renais, neurológicos e os eventos tromboembólicos venosos e arteriais (ALI MAM e SPINKLER SA, 2021). Nesse sentido, propõe-se que a infecção pelo SARS-CoV-2 predispõe a um estado pró-trombótico, responsável por elevar a possibilidade de desenvolvimento de trombose arterial, de 
trombose venosa profunda (TVP) e, consequentemente, de Tromboembolismo Pulmonar (TEP) (AZEVEDO RB, et al., 2021). Nessa perspectiva, as vasculaturas pulmonar, das pernas, do baço, do coração e do cérebro são frequentemente acometidas, o que favorece a ocorrência de falência múltipla de órgãos e acentuada morbimortalidade nos casos graves (ALI MAM e SPINKLER SA, 2021).

Partindo desse pressuposto, essa revisão narrativa objetiva analisar os mecanismos fisiopatológicos que aumentam a probabilidade de ocorrência de eventos tromboembólicos em pacientes infectados por SARSCoV-2 e a necessidade de tromboprofilaxia nessas situações.

\section{REVISÃO BIBLIOGRÁFICA}

\section{Associação entre a Covid-19 e os eventos trombóticos}

Sabe-se que a infecção pelo SARS-CoV-2 no organismo é caracterizada pela interação entre proteínas virais de superfície, proteína spike (S), e a enzima conversora de angiotensina 2 (ACE2), que promove a endocitose seguida de transcrição, tradução e replicação do genoma viral, com posterior apoptose celular, a piroptose (MOUSAVIZADEH L e GHASEMI S, 2021; CHAN NC e WEITZ JI, 2020; PRICE LC, et al., 2020). Este processo produz Padrões Moleculares Associados a Danos (DAMPs), que causam stress oxidativo no organismo e, consequentemente, produção maciça de citocinas e quimiocinas (PRICE LC, et al., 2020). Esta resposta inflamatória gera consequentes disfunções orgânicas e supressão da resposta imune adaptativa, 0 que favorece a replicação viral (BARRETTA AA, et al., 2020).

Estas citocinas, liberadas em decorrência da resposta antigênica, são estimuladas pela síntese de Interleucina-6 (IL-6), gerando, sequencialmente, aumento dos níveis séricos de Fator de Necrose Tumoralalfa (TNF- $\alpha$ ). Esta, incapaz de conter o processo infeccioso, produz uma resposta hiperinflamatória sistêmica associada a lesões teciduais, bem como maior expressão de mediadores pró-inflamatórios, como IL-1 $\beta$; IL1RA; IL-7; IL-8; IL-9; IL-10; Fator Estimulador das Colônias de Granulócitos e Macrófagos (GM-CSF); Interferon-gama (IFN- $\gamma$ ); Interferon-gama Induzido por Proteína 10 (IP10); TNF- $\alpha$ e Fator de Crescimento Endotelial Vascular (VEGF) (AARESTRUP FM, 2020).

Diante do exposto, tem-se presenciado sequelas pós-Covid-19, como comprometimento funcional, dificuldade em realizar as atividades de vida diária, modificação do desempenho profissional e prejuízo nas interações sociais, principalmente em pacientes que apresentaram a forma grave da doença (SANTANA AV, et al., 2021). Dentre as afecções encontradas, posteriormente à recuperação do quadro infeccioso, destacamse perda de peso significativa, caquexia, perda gradual das funções pulmonar e renal, doenças crônicodegenerativas do sistema nervoso e fenômenos cardiovasculares e trombóticos (ANKER MS, et al., 2021; WANG F, et al., 2020; PANITCHOTE A, et al. 2019).

Em relação aos fenômenos tromboembólicos, identificou-se que de $20 \%$ a $50 \%$ dos pacientes hospitalizados com Covid-19 manifestaram elevação do D-dímero, produto da degradação da fibrina reticulada, e do tempo de protrombina, identificando prevalência de eventos trombóticos em relação aos hemorrágicos (GÓMEZ-MESA JE, et al., 2021; CHAN NC e WEITZ JI, 2020). Estes achados estão relacionados à maior mortalidade intra-hospitalar e predizem a necessidade de elevado suporte ventilatório (GÓMEZ-MESA JE, et al., 2021). Sabe-se ainda que aproximadamente $69 \%$ dos pacientes que apresentaram Covid-19 desenvolveram tromboembolismo venoso e $71 \%$ dos pacientes que morreram de Covid-19 apresentaram critérios compatíveis com distúrbios da coagulação, principalmente a coagulação intravascular disseminada (BRANDÃO SCS, et al., 2020).

\section{Patogênese dos eventos trombóticos na Covid-19}

Os exatos mecanismos fisiopatológicos pelos quais a infecção pelo coronavírus atua no desenvolvimento da trombose ainda não são totalmente elucidados. No entanto, acredita-se que a angiotensina II, potente vasoconstritor, predispõe a um estado de hipercoagulabilidade ao elevar a produção de Fator Tecidual (FT). Nesse processo, o FT, regulado por plaquetas, por leucócitos e por células endoteliais, ativa as vias extrínseca e intrínseca da cascata de coagulação para a formação de trombina (MIESBACH W e MAKRIS M, 2020). 
Esta transforma o fibrinogênio em fibrina e ativa as plaquetas, o que corresponde a um modo de estabilização de coágulos. Esse processo ocorre de forma rápida e resistente à fibrinólise, a qual está reduzida devido ao aumento da expressão do inibidor do ativador de plasminogênio-1, e também devido ao processo inflamatório (CHAN NC e WEITZ Jl, 2020). Portanto, as coagulopatias e eventos trombóticos podem ser desencadeados mediante o aumento da angiotensina II e a redução da angiotensina 1-7, que extingue a produção de óxido nítrico e favorece a adesão de leucócitos e de plaquetas, bem como a constrição vascular e a liberação de citocinas, decorrentes da infecção pelo SARS-Cov-2 (MIESBACH W e MAKRIS M, 2020; IBA T, et al., 2020).

A cascata inflamatória, desencadeada pela presença de citocinas, induz a expressão de fator tecidual em macrófagos, o que contribui para a ativação da coagulação e interrompe a produção de trombina (GÓMEZMESA JE, et al., 2021). Esta hipercoagulabilidade decorre do envolvimento dessas moléculas na formação anômala de coágulos, na hiperativação plaquetária e na regulação negativa das vias anticoagulantes fisiológicas (MIESBACH W e MAKRIS M, 2020).

Ademais, ao considerar que o SARS-CoV-2 acarreta endotelite, que é a inflamação da camada endotelial dos vasos, compreende-se que a atividade antitrombótica do lúmen vascular encontra-se reduzida. Em condições normais, as células endoteliais regulam o tônus vascular, a permeabilidade, a adesão celular e a anticoagulação, por meio da síntese de óxido nítrico (NO) (BERNARD I, et al., 2020).

Este é responsável por impossibilitar a adesão de leucócitos e de plaquetas, a migração de células inflamatórias para a parede do vaso, a proliferação de células musculares lisas e por suprimir a apoptose e a inflamação. Na Covid-19, no entanto, essas funções estão prejudicadas, o que propicia alteração prócoagulante do lúmen vascular, formação de imunotrombose nos vasos e má circulação de órgãos afetados (IBA T, et al., 2020).

Além desses processos, a hipoxemia provocada pelo acometimento pulmonar pode gerar vasoconstrição, inflamação e, consequentemente, trombose (CHAN W e WEITZ M, 2020). Esse fato pode ser explicado pela regulação positiva do inibidor do ativador do plasminogênio e pela ativação da síntese endotelial de prócoagulantes, como o fator tecidual e de Von Willebrand, por meio da via de sinalização dependente do fator de transcrição (KATNENI UK, et al., 2020). Observa-se também maior viscosidade sanguínea nessa situação (GÓMEZ-MESA JE, et al., 2021).

Compreende-se ainda que a Coagulação Intravascular Disseminada (CID), síndrome adquirida caracterizada por ativação intravascular da coagulação, predisposta pela infecção por Covid-19, também aumenta a probabilidade de ocorrência de tromboembolismo venoso e arterial nas pessoas acometidas pela doença (SAVIOLI F e ROCHA LL, 2020). A sua patogênese é complexa e multifatorial, embora acredita-se que está relacionada ao aumento exacerbado da secreção de mediadores inflamatórios, resultante da resposta inflamatória sistêmica (DI MINNO A, et al., 2020). Infere-se também que este fenômeno está presente em cerca de $71,4 \%$ dos pacientes cujo óbito é atribuído à Covid- 19 , em comparação com $0,6 \%$ nos sobreviventes (CHAN W e WEITZ M, 2020).

Além disso, a cascata inflamatória, associada à fibrinólise prejudicada, pode ser responsável pelo surgimento de um padrão micro-trombótico, que eventualmente cursa com doenças graves, tornando-se potencialmente letal, uma vez que se resulta em microangiopatia trombótica oclusiva difusa associada a dano alveolar e a angiogênese vascular (MANOLIS AS, et al., 2021). A microangiopatia pulmonar é um fenômeno desencadeado pela Covid-19, sendo caracterizada por desenvolvimento de trombose primária dos capilares pulmonares, diferentemente do Tromboembolismo Venoso (TEV), que é gerado pela migração de um êmbolo (BRANDÃO SCS, et al., 2020). Isto posto, ressalta-se que a formação dos êmbolos e dos microtrombos pulmonares apresentam composições díspares, visto que nestes predominam plaquetas e fibrinas, enquanto os primeiros retratam as características dos trombos de origem. Dessa maneira, salienta-se, por fim, que os microtrombos capilares alveolares prevalecem nesses pacientes em comparação com os acometidos por outros vírus respiratórios, como o da Influenza A, subtipo Hemaglutinina 1 Neuroaminidase 1(H1N1) (DI MINNO A, et al., 2020). 
Nos casos pediátricos, as alterações de coagulação do Covid-19 por agressão vascular foram descritas pela Associação de Pediatras de Cuidados Intensivos do Reino Unido, pela Associação Espanhola de Pediatria e pela Sociedade Italiana de Pediatras em pacientes com características de síndrome do choque tóxico ou doença de Kawasaki, a qual consiste em uma doença inflamatória de médios vasos. A sua etiologia não é bem compreendida, embora propõe-se que possa ser desencadeada por agentes infecciosos, dentre os quais o SARS-CoV-2, e por resposta inflamatória exacerbada em crianças com predisposição genética (BRANDÃO SCS, et al., 2020). Dessa forma, nos portadores dessa condição, a infecção por Covid-19 desencadeia um quadro de endotelite com prejuízo do fluxo sanguíneo para o coração, e, consequentemente, com o desenvolvimento de trombos coronarianos (XU S, et al., 2020).

A incidência de TEV em pacientes infectados pelo SARS-CoV-2 é aproximadamente sete a oito vezes maior quando comparada com a das demais infecções respiratórias (DI MINNO A, et al., 2020). Para mais, em pacientes críticos, esse risco agrava-se, uma vez que os que fatores como imobilidade, ventilação mecânica e necessidade de cateteres centrais ampliam a possibilidade de eventos tromboembólicos em pacientes hospitalizados (SAVIOLI F e ROCHA LL, 2020). Dessa forma, constata-se que a incidência de Tromboembolismo Venoso (TEV) foi de $47 \%$ nas Unidades de Terapia Intensiva (UTI) e de $3,3 \%$ na enfermaria geral. Além de hospitalização na UTI, ressalta-se que a maior contagem de leucócitos, a maior proporção de neutrófilos/linfócitos, em associação com a elevação do D-dímero, constituem condições que propiciam o surgimento dessa condição (GÓMEZ-MESA JE, et al., 2021).

\section{Correlação entre a embolia e a Covid-19}

Considera-se, ainda, que as complicações trombóticas, observadas principalmente em pacientes graves internados em UTI, são elevadas. Dentre essas, destaca-se a embolia pulmonar aguda (EP) e arterial sistêmica, a trombose venosa profunda (TVP), o acidente vascular cerebral isquêmico e o infarto do miocárdio, tendo a EP sido referida em cerca de 18\% dos pacientes acometidos pela Covid-19 (KLOK FA, et al., 2020; DI MINNO A, et al., 2020). Diante disso, constata-se que estes pacientes se tornam vulneráveis ao desenvolvimento de falência de múltiplos órgãos devido a formação de coágulos a partir de estímulos pró trombóticos, os quais ocasionam oclusão microvascular (RIBES A, et al., 2020).

Para que esse risco seja avaliado, pode-se utilizar o D-dímero e a tomografia computadorizada. Níveis desse exame laboratorial acima de $1 \mathrm{ug} / \mathrm{ml}$ constitui indicador de pior prognóstico, posto que valores dentro da normalidade possibilitam a exclusão do risco de tromboembolismo nos pacientes estáveis com baixo risco de piora do quadro. No entanto, é necessária cautela nessa interpretação, uma vez que a especificidade desses valores é baixa (BOMPARD F, et al., 2020). A tomografia computadorizada (TC), por sua vez, permite evidenciar não apenas a presença de características hipercoaguláveis na afecção por Covid-19 grave, como também de prejuízo acentuado na circulação e perfusão pulmonar, em decorrência de angiopatia pulmonar e de trombose (MANOLIS AS, et al., 2021). Por fim, a TC com contraste também pode ser utilizada para excluir a possibilidade de tromboembolismo pulmonar (TEP). Caso o contraste não seja empregado ou não seja possível elucidar o motivo da afecção, pode-se realizar o exame de angiografia pulmonar, a fim de excluir 0 diagnóstico (LEONARD-LORENT I, et al., 2020).

Ademais, os principais fatores de risco elucidados para o desenvolvimento de TEP incluem IMC acima de $30 \mathrm{~kg} / \mathrm{m}^{2}$, faixa etária avançada, além da elevação supracitada do D-dímero e da proteína C-reativa. Todavia, não foram identificadas discrepâncias no que tange ao sexo, a etnia ou a história de doença cardiopulmonar (DI MINNO A, et al., 2020). Além do acometimento pulmonar, observou-se também o acidente vascular cerebral isquêmico como importante complicação da doença causada pelo SARS-CoV-2, originado, principalmente, pela oclusão de grandes vasos cerebrais (VOGRIG A, et al., 2021). Dessa forma, torna-se importante reconhecer a condição clínica do paciente, uma vez que doença cardiovascular prévia, bem como histórico de AVC são indicativos de pior prognóstico de Covid-19 (TREJO-GABRIEL-GALÁN JM, 2020).

\section{Tromboprofilaxia na Covid-19}

A TEV constitui um agravo à saúde que acomete em torno de 1 a cada 1.000 indivíduos adultos por ano (CUNHA MJS, et al., 2021). Tendo em vista a alta incidência de processos trombóticos venosos ou arteriais 
entre os pacientes que contraem Covid-19, torna-se necessário prevenir esses eventos, de modo a impedir maiores complicações e os desfechos desfavoráveis, gerados pelo quadro infeccioso e inflamatório (SAVIOLI F e ROCHA LL, 2020; MESA JEG, et al., 2021; PILLAI P, et al., 2021).

Diante do exposto, torna-se necessário atentar-se à trombólise e à anticoagulação, considerando-se a relação entre risco e benefício da terapia e da prevenção anti tromboembólica (PILLAI P, et al., 2021). No entanto, a despeito da profilaxia adequada, verifica-se elevada prevalência de TEV em pacientes com COVID19 (CUNHA MJS, et al., 2021). O risco de eventos tromboembólicos, analisado por meio de padrões próinflamatórios e da história prévia, além da severidade da doença devem ser avaliados para se definir a instituição de anticoagulante em dose profilática ou intermediária. Deve-se reavaliar o quadro a cada 24 a 48 horas e, caso necessário, ajustar a dosagem, como em situações de elevação de D-dímero e de sintomas graves (VIVAS D, et al., 2020).

Diante disso, a administração de heparina de baixo peso molecular pode ser considerada, de acordo com o peso, para todos os hospitalizados (VIVAS D, et al., 2020; MIESBACH W e MAKRIS M, 2020; MESA JEG, et al., 2021). À vista disso, em pacientes graves, a profilaxia deve ser instituída precocemente, uma vez que estes apresentam maior probabilidade de desenvolver eventos trombóticos com a progressão do quadro inflamatório, a despeito do baixo risco na admissão (FAJARDO JAG, et al., 2021; CARBONELL AP, et al., 2020; MIESBACH W e MAKRIS M, 2020; MESA JEG, 2021). Nesse sentido, observou-se redução da mortalidade após 7 dias de uso de enoxaparina 40-60 mg, com concomitante melhora da pressão parcial de O2 (PAO2) e diminuição da formação de microtrombos, assim como redução de trombina e eventos trombóticos venosos (VIVAS D, et al., 2020).

Recomenda-se, também, manter o uso deste medicamento por, pelo menos, um mês, uma vez que, além da ação antitrombótica, a medicação também pode contribuir para a mitigação do processo anti-inflamatório. Em casos de trombose de origem arterial ou de Acidente Vascular Encefálico (AVE), os médicos devem considerar terapia antiplaquetária (FAJARDO JAG, et al, 2021). Ademais, o uso de anticoagulante não deve estar restrito ao ambiente hospitalar, devendo o profissional de saúde avaliar a sua aplicabilidade na atenção primária à saúde, a depender da condição clínica prévia do paciente (CARBONELL AP, et al., 2020).

Contudo, no atual contexto, a inexistência de uma escala de avaliação de risco trombótico para pacientes com Covid-19 e/ou pós-Covid-19 dificulta a padronização da profilaxia para essas complicações (CONNORS JM e LEVY JH, 2020). Além disso, ressalta-se que todos os pacientes imobilizados, de baixo ou de alto risco para eventos tromboembólicos, devem se beneficiar da terapia preventiva mecânica (CARBONELL AP, et al., 2020; VIVAS D, et al., 2020). Tendo isso em vista, a fisioterapia e o uso de meias elásticas são alternativas profiláticas e terapêuticas adequadas nesse cenário, sendo que naqueles pacientes não necessitam internação hospitalar, deve-se estimular a deambulação e considerar a profilaxia de acordo com o paciente (LÓPEZ-REYES R, et al., 2021).

Cabe ressaltar que ajustes nas dosagens dos antitrombóticos devem ser previamente avaliados em caso de insuficiência renal, sendo o ritmo de filtração glomerular (RFG) o principal parâmetro a ser analisado (CONNORS JM e LEVY JH, 2020). Dessa forma, alguns fármacos, como a Dalteparina, Nadroparina e a Tinzaparina, não são recomendados para pacientes com RFG menores do que $30 \mathrm{ml} / \mathrm{min}$, devido ao significativo efeito nefrotóxico destes. Ademais, em pacientes com alto risco de hemorragia ou com sangramento ativo, o profissional deve considerar apenas a tromboprofilaxia mecânica, a fim de evitar o desencadeamento ou o agravamento do sangramento e, consequentemente, o choque hipovolêmico (CARBONELL AP, et al., 2020). O uso desta medicação também está contraindicada se a contagem de plaquetas estiver abaixo de 25000/ml (VIVAS D, et al., 2020).

Além disso, devido à interação medicamentosa entre anticoagulantes orais, antivirais e antagonistas de vitamina $\mathrm{K}$, recomenda-se o uso de heparina não fracionada ou de baixo peso molecular, associado ou não à profilaxia mecânica (CARBONELL AP, et al., 2020; MESA JEG, et al., 2021). No caso de pacientes diagnosticados com Covid-19 que apresentam evento trombótico concomitante, seja na terapia intensiva ou na enfermaria, o uso da heparina não-fracionada é preferível (MIESBACH W e MAKRIS M, 2020; MESA JEG, et al., 2021). Por fim, os anticoagulantes orais, como o rivaroxaban, apixaban e ticagrelor, podem apresentar 
concentração aumentada no organismo, e por isso não devem ser indicados em situações de uso conjunto com antirretrovirais, como lopinavir e ritonavir (CONNORS JM e LEVY JH, 2020).

\section{CONSIDERAÇÕES FINAIS}

Observa-se que o SARS-CoV-2, por sua aparente conformação estrutural e genética, promove uma reação imune predisponente à uma resposta inflamatória rápida e, em alguns casos, exacerbada e sistêmica, acometendo, de forma considerável, o sistema cardiovascular. Diante disso, as teorias propostas consideram que a patogênese dos eventos trombóticos na Covid-19 relaciona-se às interações entre as proteínas virais e as células do indivíduo. Destaca-se, portanto, a ativação da cascata de coagulação, inicialmente provocada pela angiotensina II, e a expressão de fator tecidual em macrófagos resultando na formação de trombina, a endotelite, a hipoxemia e a coagulação intravascular disseminada. Nesse cenário, a tromboprofilaxia assume suma importância na prevenção de desfechos desfavoráveis com possíveis intervenções em diferentes âmbitos. Nessa perspectiva, a heparina de baixo peso molecular, principal droga de escolha em pacientes hospitalizados, associada à terapia mecânica, destaca-se como terapêutica benéfica na prevenção de processos trombóticos.

\section{REFERÊNCIAS}

1. AARESTRUP FM. Imunopatologia da COVID-19 e suas implicações clínicas. Arquivos de asma, alergia e imunologia, 2020; 4(2): 172-180.

2. ALI MAM, SPINLER SA. COVID-19 and thrombosis: From bench to bedside.Trends Cardiovasc Med, 2021,31 (3): 143-160.

3. ANKER MS, et al. Weight loss, malnutrition, and cachexia in COVID-19: facts and numbers. J Cachexia Sarcopenia Muscle, 2021; 12(1): 9-13.

4. AZEVEDO RB, et al. Covid-19 and the cardiovascular system: a comprehensive review. J Hum Hypertens, 2021; 35(1): 4-11.

5. BARRETTA AA, et al. Propolis and its potential against SARS-CoV-2 infection mechanisms and COVID-19 disease. Biomed Pharmacother, 2020; 131.

6. BELTRÁN MB, et al. Initial symptomatology of SARS-CoV-2 infection in healthcare personnel: What have we learned? Aten Primário, 2021, 53(2).

7. BERNARD I, et al. Endothelium Infection and Dysregulation by SARS-CoV-2: Evidence and Caveats in COVID-19. Viruses, 2020; 13(1): 29.

8. BHARGAVA P, et al. Acute Pulmonary Embolism and COVID-19. Radiology, 2020; 297(3).

9. BOMPARD F, et al. Pulmonary embolism in patients with COVID-19 pneumonia. Eur. Respir. J, $2020 ; 56$ (1).

10. BRANDÃO SCS, et al. COVID-19 grave: entenda o papel da imunidade, do endotélio e da coagulação na prática clínica. J Vasc Bras, 2020.

11. BRASIL. Ministério da Saúde. Painel Coronavírus. Brasília, 2021. Disponível em: <https://covid.saude.gov.br/>. Acesso em: 5 jun. 2021.

12. CARBONELL AP, et al. COVID-19 y tromboprofilaxis: recomendaciones para nuestra práctica clínica en Atención Primaria. Semergen, 2020; 46 (7): 479-486.

13. CHAN NC, WEITZ, JI. COVID-19 coagulopathy, thrombosis, and bleeding. Thrombosis and hemostasis, 2020; 136(4).

14. CONNORS JM, LEVY JH. COVID-19 and its implications for thrombosis and anticoagulation. Blood, 2020; 135 (23):2033-2040.

15. CUNHA MJS, et al. Incidence, diagnosis, treatment methods, and outcomes of clinically suspected venous thromboembolic disease in patients with COVID-19 in a quaternary hospital in Brazil. J. Vasc. Bras, 2021; 20.

16. DI MINNO A, et al. COVID-19 and Venous Thromboembolism: A Meta-analysis of Literature Studies. Semin Thromb Hemost, 2020; 46(7): 763-771.

17. FAJARDO JAG, et al. Mortalidad de los pacientes covid-19 com complicaciones trombóticas. Med Clin, 2021 ; 156 (3): 112-117.

18. GÓMEZ-MESA JE, et al. Thrombosis and Coagulopathy in COVID-19. Curr Probl Cardiol, 2021; 46 (3).

19. IBA T, et al. The coagulopathy, endotheliopathy, and vasculitis of COVID-19. Inflamm. Res, 2020; 69: 1181-1189.

20. KATNENI UK., et al. Coagulopathy and Thrombosis as a Result of Severe COVID-19 Infection: A Microvascular Focus. Thrombosis and Hemostasis, 2020; 120(12).

21. KLOK FA, et al. Incidence of thrombotic complications in critically ill ICU patients with COVID-19. Thromb Res, 2020; 191: 145-147.

22. LEONARD-LORANT I, et al. Acute Pulmonary Embolism in COVID-19 Patients on CT Angiography and Relationship to D-Dimer Levels. Radiology 2020, 2020.

23. LOBO AP, et al. COVID-19 epidemic in Brazil: Where are we at? Int J Infect Dis, 2020; 97: 82-385. 
24. LÓPEZ-REYES R; et al. Thrombotic Risk and Covid-19: Review of Current Evidence for a Better Diagnostic and Therapeutic Approach. Arch bronconeumol, 2021; 57: 55-64.

25. MANOLIS AS, et al. Infecção por COVID-19: coagulopatia viral macro e microvascular e tromboembolismo / manejo profilático e terapêutico. J Cardiovasc. Pharmacol. Ther, 2021; 26 (1): 12-24.

26. MESA JEG, et al. Trombosis and coagulopathy in COVID-19. Curr Probl Cardiol, 2021; 46 (3).

27. MIESBACH W, MAKRIS M. COVID-19: Coagulopathy, Risk of Thrombosis, and the Rationale for Anticoagulation. Clinical and Applied Thrombosis/Hemostasis, 2020; 26: 1-7.

28. MOUSAVIZADEH L, GHASEMI S. Genotype and phenotype of COVID-19: Their roles in pathogenesis. J Microbiol Immunol Infect, 54 (2): 159-163.

29. PANITCHOTE A, et al. Factors associated with acute kidney injury in acute respiratory distress syndrome. Annals of Intensive Care, 2019; 74

30. PILLAI P, et al. COVID-19 and Major Organ Thromboembolism: Manifestations in Neurovascular and Cardiovascular Systems. J Stroke Cerebrovasc Dis, 2021; 30 (1).

31. PRICE LC, et al. Thrombosis and COVID-19 pneumonia: the clot thickens!. Eur Respir J, 2020; 56.

32. RIBES A, et al. Thromboembolic events and Covid-19. Adv Biol Regul, 2020; 77.

33. SANTANA AV, et al. Reabilitação pulmonar pós-Covid-19. J Bras Pneumol, 2020; 47(1).

34. SAVIOLI F, ROCHA LL. Perfil de coagulação em pacientes com COVID-19 grave: o que sabemos até aqui? Rev. Bras. Ter.Intensiva, 2020; 32 (2): 197-199.

35. TREJO-GABRIEL-GALÁN JM. Stroke as a complication and prognostic factor of COVID-19. Neurologia, $2020 ; 35$ (5): 318-322.

36. VIVAS D, et al. Recommendations on antithrombotic treatment during the Covid-19 pandemic. Positions statement of the working group on cardiovascular thrombosis of the spanish society of cardiology. Rev Esp Cardiol, 2020; 73 (9): 749-757.

37. VOGRIG A, et al. Stroke in patients with COVID-19: Clinical and neuroimaging characteristics. Neurosci Lett, 2021; 743.

38. WANG F, et al. Long-Term Respiratory and Neurological Sequelae of COVID-19. Med Sci Monit, $2020 ; 26$.

39. XU S, et al. COVID-19 and Kawasaki disease in children. Pharmacol Res, 2020; 159. 